\title{
Peso e altura auto-referidos: um breve relato da literatura
}

\author{
Antônio Felipe Corrêa Marangon ${ }^{1}$ \\ Luis Gabriel de Melo Fernandes ${ }^{2}$ \\ Priscilla Marcondelli ${ }^{3}$
}

\begin{abstract}
RESUMO - O método de peso e altura auto-referidos é comumente utilizado em pesquisas com elevado número de participantes em decorrência da praticidade e do baixo custo. Entre os estudos realizados, tanto entre as mulheres quanto entre os homens, independentemente de serem adultos ou adolescentes, há a tendência de subestimação do peso, mais acentuada entre as mulheres. No entanto, indivíduos com distúrbios alimentares demonstram maior precisão no auto-referimento do peso. Em relação à altura, tanto homens quanto mulheres - adultos e adolescentes - tendem a superestimar a altura, porém essa tendência não ocorre em indivíduos com distúrbios alimentares.
\end{abstract}

Palavras-chave: peso, altura, auto-referimento, distúrbios alimentares.

\section{Self-reporting weight and heigh: a brief revision}

\begin{abstract}
The method weight and height self-reported usually is used in research of raised number of participants due to its praticidade and low cost. Among the carried through studies the respect, as much enters the women how much between the men, independent to be adult or adolescent, it has a trend of subestimation of the weight, being, it enters the women more accented. However, individuals with eating disorders demonstrate to greater precision in the selfreport of the weight. In relation to the height, as many men how much women adult and adolescent - tend to overestimate its height, however this trend does not occur in individuals eating disorders.
\end{abstract}

Key words: weight, height, self-report, eating diseases.

${ }^{1}$ Graduado em Educação Física e Nutrição; especialista em Fisiologia do Exercício e Nutrição para o Fitness e o Alto Rendimento; mestrando em Educação Física antonio.felipe@ uniceub.br

${ }^{2}$ Graduando em Nutrição - UniCEUB. E-mail: lgabriel2@ terra.com.br

${ }^{3}$ Graduada em Nutrição; especialista em Fisiologia do Exercício e mestre em Nutrição Humana. 
O método classificado como peso e altura auto-referidos refere-se à coleta dados de forma simples, eficiente e não invasiva. Além de ser prático, é acessível, não despende tempo e pode ser utilizado em populações com elevado número de participantes ${ }^{1}$. Para realizar o procedimento, primeiramente, o entrevistador pergunta o peso e a altura do indivíduo e, em seguida, submete-o à avaliação antropométrica para confirmar o peso e a altura, constatando a diferença entre o peso e altura auto-referidos e os medidos.

Após discutir as implicações das diferenças encontradas no peso medido e no auto-referido, é importante notar suas possíveis causas. Uma delas pode ser o desconhecimento do próprio peso. Outra pode ser relacionada com a imagem pessoal que, independentemente de conhecer a precisão do seu peso, a pessoa tem idéia errônea da sua imagem corporal. Ademais, esse problema pode relacionar-se à informação sobre o peso: mesmo se a pessoa conhece precisamente seu peso, muitos fatores, como normas culturais ou psicologia pessoal, induzem ao relato errado na entrevista ${ }^{2}$.

Outras razões podem estar relacionadas a erros no auto-referimento de peso, como, por exemplo, os indivíduos podem relatar seu peso mais baixo pela manhã, em jejum e sem roupas. Entretanto, os investigadores que realizam mensuração de peso e/ ou estatura fazem-na ao longo do dia, com roupas e, às vezes, logo após uma refeição. Assim, o valor obtido na mensuração não coincide com o real pelo peso atribuído às vestimentas ${ }^{3}$. Para o peso auto-referido, ocorre a tendência que parece ser a de imagem corporal culturalmente desejável: indivíduos com sobrepeso subestimam o peso, enquanto indivíduos abaixo do peso o superestimam, na tentativa de enquadrá-lo nos padrões determinados culturalmente ${ }^{4}$.

Embora os valores medidos e os auto-referidos estejam fortemente co-relatados, significativas subestimações de peso e superestimações de altura foram observadas por Niedhammer e colaboradores, tanto para homens quanto para mulheres. Esses dados conduzem à subestimação do Índice de Massa Corporal IMC, levando à prevalência de sobrepeso. Uma explicação para esse fator foi a medida do IMC: quanto maior a medida do IMC, maior a subestimação do peso e a superestimação da altura em ambos os $\operatorname{sexos}^{5}$.

A superestimação de altura aumenta significativamente com a idade, talvez pelo fato de as pessoas mais velhas passarem mais tempo sem medir a altura,

\footnotetext{
${ }^{1}$ NIEDHAMMER et al., 2000.

${ }^{2}$ SCHMIDT et al., 1993.

${ }^{3}$ STEWART et al., 1987.

${ }^{4}$ SCHMIDT et al., 1993.

${ }^{5}$ REED \& PRICE, 1998.
} 
considerando, também, a sua diminuição como parte do processo natural da ida$\mathrm{de}^{6}$. Fatores socioeconômicos, como nível educacional ocupacional, podem estar associados a erros no referimento do peso e da altura ${ }^{7}$.

A superestimação da altura é comum, pois a maioria dos adultos não se mede com freqüência, o que pode ser verdadeiro para adultos mais velhos que sofrem o encolhimento por processos de catabolismo ósseo, achatamento dos espaços vertebrais e alterações anatômicas, como a cifose. O peso auto-referido, em contrapartida, é influenciado por "normas" sociais de magreza, levando os indivíduos a expressar um peso socialmente desejável. Outra explicação também ajuda a mostrar a tendência de mulheres com sobrepeso subestimarem o peso mais que homens com sobrepeso, e mulheres mais jovens subestimarem o peso mais que mulheres mais velhas. Isso ocorre porque as expectativas sociais de magreza são mais pronunciadas em mulheres que em homens, particularmente em mulheres mais jovens, o que sugere que elas sejam influenciadas pela preocupação com as normas sociais ${ }^{8}$.

O relato do peso pode ser visto como um processo de auto-apresentação social. Indivíduos com maior peso corporal nos quais ocorre discrepância pela percepção de um peso ideal são mais suscetíveis a subestimá-lo9 ${ }^{9}$ Indivíduos com excesso de peso corporal e preocupados com peso, entre aqueles pacientes com distúrbios alimentares ou que seguem dietas, podem ser mais suscetíveis a distorcer o peso que aqueles que não têm preocupação com a forma corporal ${ }^{10}$. Esse pressuposto por subestimação de peso está associado a maior restrição alimentar, busca por magreza e medo de gordura ${ }^{11}$.

\section{Mulheres}

Mulheres são mais suscetíveis a subestimar o peso, diminuindo em até $5 \mathrm{~kg}$, quando comparadas com os homens ${ }^{12}$. Estudo realizado por Reed \& Price (1998) demonstrou que mulheres extremamente obesas superestimam a altura em média de $0,9 \mathrm{~cm}$ e subestimam o peso em média de $0,8 \mathrm{~kg}$. Os falsos dados estimam um resultado em valores de IMC menores que os medidos em cerca de 0,8 unidades de IMC. Neste mesmo estudo, foi feita avaliação para saber a precisão da estimativa de peso e altura que um informante relatava de sua família e constatou-se que

\footnotetext{
${ }^{6}$ STEWART et al., 1987.

${ }^{7}$ NIEDHAMMER et al., 2000.

${ }^{8}$ LARSON, 2000.

${ }^{9}$ CASH et al., 1989.

${ }^{10}$ KLESGES et al., 1991.

${ }^{11}$ CASH et al., 1992.

${ }^{12}$ STRAUSS, 1999.
} 
há diferenças na correlação entre auto-referimento e a estimativa do informante para mulheres obesas.

Indivíduos que tinham mulheres obesas na família superestimaram a altura em média $0.8 \mathrm{~cm}$ e subestimaram o peso em média de $6,9 \mathrm{~kg}$, os quais resultaram em subestimação do valor do IMC por volta de 2,9 unidades. Foi constatado que as mulheres, de maneira geral, quando informantes, estimaram o peso, em média, 3,5 $\mathrm{kg}$ a menos do que os valores medidos.

Entre as mulheres avaliadas em estudo feito por Schmidt e colaboradores (1993), a subestimação informada sobre o peso foi de $1,2 \mathrm{~kg}$, enquanto aquelas com peso normal subestimaram-no em $0.07 \mathrm{~kg}$. As mulheres com sobrepeso subestimaram-no em $1 \mathrm{~kg}$, e as obesas, em 1,2 kg. Em contrapartida, mulheres obesas são mais precisas no que diz respeito a estimar seu peso que mulheres magras ${ }^{13}$.Em outro estudo realizado por Stewart (1987) e colaboradores, concluiu-se que as mulheres divergem tanto entre os valores de peso auto-referidos e os valores medidos quanto entre os valores de altura auto-referidos e os valores medidos.

Em contraste com os dados apresentados para os homens, largas proporções de mulheres com IMC aceitáveis estão na categoria de risco baseado na circunferência da cintura. Entretanto, 9\% daquelas com idade entre 18 e 39 anos, $30 \%$ entre 40 e 59 anos e $39,1 \%$ entre 60 e 78 anos estavam na categoria de risco baseado na circunferência da cintura. Comparando a prevalência de risco baseado somente na medida do IMC, a prevalência de sobrepeso baseado na combinação de medidas, IMC ( $>25 \mathrm{~kg} / \mathrm{m} 2)$ e circunferência do quadril $(>80 \mathrm{~cm})$, foi $6,9 \%$, $18,1 \%, 17,6 \%$ e $13,9 \%$ maior para mulheres entre 18 e 39,40 e 59 e 60 e 78 anos, respectivamente. A prevalência de sobrepeso baseado na combinação do IMC auto-referido e com a circunferência da cintura é maior se comparada com a prevalência baseada somente no IMC para todos os grupos de idade entre as mulheres. Para mulheres com idade entre 60 e 78 anos, a prevalência de sobrepeso quase duplica quando a circunferência da cintura também é considerada ${ }^{14}$. Estudos demonstram que, quanto maior for a circunferência da cintura, maiores os riscos relacionados com a obesidade, como, por exemplo, hipertensão arterial sistêmica, dislipidemias, diabetes, sendo o valor limite para mulheres de $88 \mathrm{~cm}^{15}$.

Niedhammer e colaboradores (2000) concluíram que, quanto maior o nível educacional, menor a superestimação de altura. Entre as mulheres, altos níveis ocupacionais foram associados com aumento da superestimação da altura, e mulheres com níveis ocupacionais mais baixos foram mais suscetíveis a subestimar

\footnotetext{
${ }^{13}$ REED \& PRICE, 1998.

${ }^{14}$ BOOTH et al., 2000.

${ }^{15}$ NIEDHAMMER et al., 2000.
} 
seu peso. Outra associação foi feita com mulheres que tomavam medicamentos para doenças cardiovasculares e que foram mais suscetíveis a superestimar a altura. Além disso, mulheres altas foram mais suscetíveis a subestimar o peso. Quanto maior for a medida do IMC, maior a subestimação do peso e a superestimação da altura $^{16}$.

Estudo realizado por Larson (2000) constatou que mulheres com sobrepeso tendem a subestimar o peso mais que homens com sobrepeso, e mulheres mais jovens subestimam o peso mais que mulheres mais velhas. Além disso, mulheres com menor peso corporal tendem a superestimar o peso. A altura auto-referida, em contrapartida, é freqüentemente superestimada em populações de adultos mais velhos, e os homens, geralmente, tendem a superestimar mais que as mulheres. Nesse mesmo estudo, $25 \%$ da amostra total das mulheres com média de $71,2 \mathrm{~kg}$ subestimaram seu peso, em média, $3,9 \mathrm{~kg}$, enquanto $25 \%$ das mulheres com 49,5 $\mathrm{kg}$, em média, superestimaram $0,6 \mathrm{~kg}$.

Ao contrário do grupo dos homens, as mulheres subestimaram o peso em todas as categorias, porém isso tende a diminuir com o aumento da idade. A diferença média entre o peso medido e o auto-referido foi significantemente maior em mulheres que em homens $(0,85$ e 0,54 kg), conseqüentemente, é uma média maior entre o IMC medido e o auto-referido $(0,44 \text { e } 0,29 \mathrm{~kg} / \mathrm{m} 2)^{17}$. Em média, o IMC relatado é subestimado em grau maior por mulheres autodescritas com sobrepeso do que entre aquelas abaixo do peso ou no peso normal ${ }^{18}$.

\section{Homens}

Entre os homens, a subestimação informada sobre o peso é de $1,2 \mathrm{~kg}$, enquanto aqueles com o peso normal subestimam-no peso em $0,42 \mathrm{~kg}$. Os homens com sobrepeso subestimam-no em $0,22 \mathrm{~kg}$, e os obesos, em 1,4 kg. Nesse mesmo sentido, os homens relatam o peso de $0,67 \mathrm{~kg}$ a menos que relatado pelas mulheres ${ }^{19}$.

A subestimação de peso aumenta de acordo com o aumento da idade dos homens. Em particular, homens que tomam medicamentos para combater doenças cardiovasculares são mais suscetíveis a subestimar o peso. Homens magros são mais suscetíveis a superestimá-lo ${ }^{20}$, e somente homens obesos tendem a subesti-

\footnotetext{
${ }^{16}$ NIEDHAMMER et al., 2000.

${ }^{17}$ NIEDHAMMER et al., 2000.

${ }^{18}$ DAVIS \& GERGEN, 1994.

${ }^{19}$ SCHMIDT et al., 1993.

${ }^{20}$ NIEDHAMMER et al., 2000.
} 
mar o peso atual ${ }^{21}$. Homens não fumantes subestimam o peso mais que homens fumantes ${ }^{22}$.

Assim como no grupo das mulheres, quanto maior for a medida do IMC, maior a subestimação do peso e a superestimação da altura. Em contrapartida, menores valores de IMC são associados com a superestimação do peso ${ }^{23}$. No grupo dos homens, também há divergência tanto entre os valores de peso auto-referidos e os valores medidos quanto entre os valores de altura auto-referidos e os valores medidos ${ }^{24}$.

Reed \& Price (1998) realizaram estudo onde foi feita avaliação para saber a precisão da estimativa de peso e altura que um informante relatava de sua família. Neste sentido, foi constatado que os homens, quando informantes, subestimaram o peso na média de $5,3 \mathrm{~kg}$ a menos que o peso medido.

Homens com IMC abaixo de $25 \mathrm{~kg} / \mathrm{m}^{2}$ superestimam o peso, enquanto indivíduos com sobrepeso e obesos são mais susceptíveis a subestimá-lo ${ }^{25}$. Entre os homens com idade menor que 60 anos, a prevalência de sobrepeso baseado na combinação do IMC auto-referido com a medida da circunferência da cintura é ligeiramente maior que a prevalência de sobrepeso baseado no IMC auto-referido. Entretanto, entre os homens com idade superior a 60 anos, a prevalência de sobrepeso baseado na combinação do IMC auto-referido e com a circunferência da cintura é, aproximadamente, $20 \%$ maior que a prevalência baseada somente na medida do $\mathrm{IMC}^{26}$. Não há diferenças no grau de subestimação de peso em homens de diferentes $\operatorname{raças}^{27}$.

Em relação à altura auto-referida, os homens superestimam-na mais que as mulheres, porém, quanto maior o nível educacional, menor a superestimação de altura. Em contrapartida, altos níveis ocupacionais estão associados ao aumento na superestimação ${ }^{28}$. STEWART et al. constataram que a superestimação da altura é maior em homens que em mulheres, $2,14 \mathrm{~cm}$ comparado com 1,60 cm, e, em ambos os sexos, há aumento significativo de acordo com a idade.

Normalmente, quando o pai auto-refere a altura, superestima-a mais que outros membros da família. Similarmente, informantes da família superestimam a altura dos pais mais que de outros membros da família. Uma explicação parcial para isso é que o pai é considerado o chefe da família, exercendo, portanto, papel crucial ${ }^{29}$.

${ }^{21}$ STRAUSS, 1999.

22 STEWART et al., 1987.

${ }^{23}$ NIEDHAMMER et al., 2000.

${ }^{24}$ STEWART et al., 1987.

${ }^{25}$ NIEDHAMMER et al., 2000.

${ }^{26}$ BOOTH et al., 2000.

${ }^{27}$ STRAUSS, 1999.

${ }^{28}$ NIEDHAMMER et al., 2000.

${ }^{29}$ REED \& PRICE, 1998. 


\section{Adolescentes}

Como já mencionado, geralmente, adultos tendem a subestimar o peso e a superestimar a altura quando auto-referidos, porém poucos estudos têm investigado essa relação em populações de adolescentes, em amostras específicas. Dessas populações jovens, o peso tende a ser subestimado (entre 0,8 e $2 \mathrm{~kg}$ ) e a altura superestimada (entre 0,5 e $1,5 \mathrm{~cm}$ ). Os fatores encontrados para essa relação imprecisa dos valores são a idade, a altura atual, o peso e o IMC. Embora o desenvolvimento da puberdade esteja associado a rápidas mudanças na altura e no peso, o interesse pela imagem corporal e por prática de exercício em pessoas jovens pode ser a preocupação em adolescentes pelos efeitos do crescimento e do desenvolvimento, mesmo sendo considerado preditor do relato de altura ou peso impreciso nos adolescentes ${ }^{30}$.

Há relação linear entre os estágios de Tanner e a precisão da altura autoreferida, a qual progride com considerável subestimação de altura para estudantes que iniciam a puberdade ( $1^{\circ}$ ou $2^{\circ}$ estágio de Tanner) para a estimação precisa para estudantes que completaram as mudanças da puberdade ( $5^{\circ}$ estágio de Tanner). Quando ocorre aumento no nível de exercício, os estudantes progridem da subestimação da altura $(\mathrm{em} 1,4 \mathrm{~cm})$ para a precisão da estimativa da altura $(0,1 \mathrm{~cm}$ maior que a altura medida). A relação entre os estágios de Tanner e a precisão do peso auto-referido não está clara como na altura auto-referida. Estudantes de todos os estágios de puberdade subestimaram o peso. Entretanto, estudantes no estágio 3 foram menos precisos (subestimação de $1,6 \mathrm{~kg}$ ) que estudantes que completaram a puberdade (Estágio 5)(subestimação de $0,5 \mathrm{~kg})^{31}$.

Em particular, garotas são mais suscetíveis a subestimar o peso, comparadas com garotos, que são mais suscetíveis a superestimar a altura. Em média, os garotos subestimaram o peso em $1,8 \mathrm{~kg}$, e as garotas, em $1,9 \mathrm{~kg}$, enquanto a altura foi superestimada pelos garotos em média de $2,3 \mathrm{~cm}$ e pelas garotas, de $0,8 \mathrm{~cm}^{32}$.

Diferenças entre o peso atual e o peso auto-referido é significativamente maior em crianças obesas, quando comparadas a crianças não-obesas (4.6 +- 7.8 $\mathrm{kg}$ vs $0.2+-5.3 \mathrm{~kg})^{33}$. Para adolescentes mulheres, o estágio do desenvolvimento da puberdade, desde a menarca, e a atividade física são preditores da precisão de altura auto-referida, peso corporal e IMC (calculado por valores auto-referidos). Garotas que têm desenvolvimento da puberdade mais cedo são mais suscetíveis à maior subestimação da altura. É possível que, no período pré-menarca, não es-

\footnotetext{
${ }^{30}$ ABRAHAM et al., 2004.

${ }^{31}$ ABRAHAM et al., 2004.

${ }^{32}$ GIACCHI et al., 1998.

${ }^{33}$ STRAUSS, 1999.
} 
tejam atentas ao aumento na estatura e, mais adiante, subestimem a altura. Após a menarca, quando o estirão do crescimento foi completado, os valores da altura têm estimativa mais precisa. Garotas que têm dois anos pós-menarca podem perceber a altura ideal e, mais adiante, superestimá-la ${ }^{34}$.

Adolescentes entre 12 e 14 anos de idade subestimam ligeiramente a altura, enquanto aqueles entre 18 e 19 anos ligeiramente superestimam-na ${ }^{35}$. O IMC baseado nos auto-referimentos é superestimado por estudantes que têm baixo peso. Para outros estudantes, a subestimação do IMC aumenta quando o atual IMC também aumenta. Estudantes com baixo peso (menor que $46.4 \mathrm{~kg}$ ) superestimam o IMC em 0,6, enquanto estudantes com excesso de peso (maior que $64.6 \mathrm{~kg}$ ) subestimam o IMC em 1,1. Estudantes no período da pré-puberdade superestimam os valores do IMC em 0,9, e as que alcançam a menarca na previsão de 12 meses são bastante precisas, superestimam em 0,1 . Em contrapartida, os que se encontram fora desses estágios têm ligeira superestimação no IMC, em 0,4. Os estudantes mais precisos são os que praticam atividade física. Entretanto, há progressão de ligeira superestimação do IMC por estudantes com nível baixo de atividade física, em 0,3, para superestimação entre aquelas com nível alto de atividade, também em $0,3^{36}$. Para ambos os sexos, adolescentes com IMC baixo superestimam o peso, e os com IMC alto superestimam-no ${ }^{37}$.

\section{Indivíduos com distúrbios alimentares}

Notou-se significativa relação entre subestimação do peso e nível de restrição alimentar na qual indivíduos que seguem dietas subestimam mais seu peso, comparado com indivíduos que não o fazem. Se o relato do peso for visto como processo de auto-avaliação social, pode ser motivo para indivíduos que seguem dietas subestimarem o peso e mostrarem auto-apresentação de serem mais magros do que realmente são. Entretanto, isso pode também refletir a auto-apresentação projetada para fazer sentir-se melhor, impulsionando valor pessoal. Tanto indivíduos que seguem dietas quanto indivíduos que não o fazem mostram tendência a subestimar o peso. Porém, a subestimação é mais pronunciada em pessoas que seguem dietas, o que parece refletir preocupação social para ter um corpo magro $^{38}$.

Uma hipótese para pacientes com distúrbios alimentares pode ser a suscetibilidade para distorcer o peso corporal mais que indivíduos sadios, confe-

\footnotetext{
${ }^{34}$ ABRAHAM et al., 2004.

${ }^{35}$ DAVIS \& GERGEN, 1994.

${ }^{36}$ ABRAHAM et al., 2004.

${ }^{37}$ DAVIS \& GERGEN, 1994.

${ }^{38}$ MCCABE et al., 2000.
} 
rindo observação clínica em pesquisa que demonstra tenderem os primeiros a superestimar o tamanho corporal. Pacientes com quadro de anorexia nervosa (AN) e bulimia nervosa (BN) são precisos no relato do peso, embora pacientes com AN tenham ligeira tendência a superestimá-lo, e pacientes com BN, a subestimálo. Porém, é importante notar que a ligeira tendência é por volta de $1 \mathrm{~kg}$ do peso atual ${ }^{39}$.

Indivíduos com bulimia nervosa têm grande interesse no peso, o que expressa aumento na precisão do auto-referimento. Esse efeito parece ser mais acentuado pelo intenso desejo de ser magro, o que pode aumentar a frequiência com que esses indivíduos se pesam. Em média, 50\% dos indivíduos com bulimia nervosa pesam-se mais que uma vez por semana, o que acontece, em média, com $20 \%$ das mulheres saudáveis ${ }^{40}$. Indivíduos que comem compulsivamente podem ser suscetíveis a subestimar o peso de acordo com o desejo por ser magro ou por medo de gordura. De antemão, isso pode ter uma precisão incomum em razão do interesse pelo peso $^{41}$.

Um achado para as associações entre o grau de preocupação com o peso e a precisão do auto-referimento parece correr para direções opostas em dois grupos. Em indivíduos sadios, quanto maior o grau de preocupação com o peso, maior é o grau de subestimação do peso. Em indivíduos com bulimia nervosa, quanto maior o grau de preocupação com o peso, mais preciso é o auto-referimento. Porém, em indivíduos com bulimia nervosa, o aumento dos níveis de preocupação com o peso parece estar associado ao aumento na tendência de subestimação do peso. Entretanto, a diferença entre o peso atual e o desejável em indivíduos com bulimia nervosa é muito maior que a observada em indivíduos saudáveis ${ }^{42}$.

Não há significativas diferenças entre a altura auto-referida e a atual entre pacientes com anorexia nervosa, bulimia nervosa, indivíduos que seguem dietas e indivíduos que não seguem dieta. Todos têm tendência a superestimar a altu$\mathrm{ra}^{43}$. Estudo realizado por DOLL \& FAIRBURN mostrou que indivíduos sadios superestimaram a altura em $0,18 \mathrm{~cm}$. Indivíduos com bulimia nervosa não mostraram tendência para superestimar a altura. Em relação ao peso, indivíduos sadios subestimaram o peso em média de $1,01 \mathrm{~kg}$, resultando em subestimação do IMC em 0,41 unidades. Em contraste, em indivíduos com bulimia nervosa, não foi significativa a subestimação do peso, e o IMC calculado com base em valores autoreferidos não foi significativamente diferente do verdadeiro IMC.

\footnotetext{
${ }^{39}$ MCCABE et al., 2000.

${ }^{40}$ DOLL \& FAIRBURN, 1998.

${ }^{41}$ MCCABE et al., 2000.

${ }^{42}$ DOLL \& FAIRBURN, 1998.

${ }^{43}$ MCCABE et al., 2000.
} 


\section{Conclusão}

O relato de peso e altura é facilmente obtido, o que torna apropriada a utilização em pesquisas que buscam avaliar obesidade e comportamentos associados. No entanto, não é seguro para pesquisas longitudinais em que medidas mais precisas são necessárias. O relato de peso e altura não é recomendado para uso em estudos de obesidade co-relatadas com morbidades, como hipertensão ou hipercolesterolemia, em que é mais apropriada a utilização de métodos antropométricos ${ }^{44}$.

A validez do peso auto-referido é suficiente para permitir a utilização em pesquisas em que ocorre a prevalência de obesidade e outras categorias de peso, porém a validez do peso e altura auto-referidos deve ser sempre questionada, e a coleta desses dados deve ser evitada sempre que possível ${ }^{45}$.

${ }^{44}$ STRAUSS, 1999.

${ }^{45}$ SCHMIDT et al., 1993.

\section{Referências}

ABRAHAM, S. A.et al. "Predictors of the Accuracy of Self-Reported Height and Weight in Adolescent Female School Students”.In: Wiley Periodicals Inc., 36, pp. 76-82.

BOOTH, M. L.et al. "The relationship between body mass index and waist circumference: implications for estimates of the population prevalence of overweight". In: International Journal of Obesity, 2000, 24, pp. 1059-1061.

DAVIS, H. \& GERGEN, P. J. “The weights and heights of Mexican-American adolescents: the accuracy of self-reports". In: American Journal of Public Health, 1994, 3, pp. 459-462.

DOLL, H. A. \& FAIRBURN, C. G. "Heightened accuracy of self-reported weight in bulimia nervosa: a useful cognitive 'distortion'”. In: John Wiley \& Sons INC., . 1998, 24, pp. 267-273.

GIACCHI, M., MATTEI, R., ROSSI, S. Correction of the self-reported BMI in a teenage population. International Journal of Obesity, . 1998, 22, pp. 673-677.

LARSON, M. R. "Social desirability and self-reported weight and height". In: International Journal of Obesity, . 2000, 24, pp. 663-665.

MCCABE, R. E. et al. "Eating disorders, dieting, and the accuracy of self-reported weight". In: John Wiley \& Sons Inc., 2000, 29, pp. 59-64.

NIEDHAMMER, I. et al. "Validity of self-reported weight and height in the French GAZEL 
cohort". In: International Journal of Obesity, 2000, 24, pp. 1111-1118.

REED, D. R. \& PRICE, R. A. "Estimates of the heights and weights of family members: accuracy of informant reports". In: International Journal of Obesity, 1998, 22, pp. 827-835.

SCHMIDT, M. I. et al. "Validity of self-reported weight - A study of urban brazilian adults". In: Rev. Saúde Pública, 1993, 27, pp. 271-276.

STEWART, A. W. et al. "Underestimation of relative weight by use of self-reported height and weight". In: American Journal of Epidemiology, 1987, 125, pp. 122-126.

STRAUSS R. S. "Comparison of measured and self-reported weight and height in a crosssectional sample of young adolescents". In: International Journal of Obesity, 1999, 23, pp. 904-908. 
Para publicar na revista Universitas - Ciências da Saúde, encaminhe seu artigo original para:

Campus do Centro Universitário de Brasília UniCEUB, SEPN 707/907, Bloco 9, Faculdade de Ciências da Saúde, CEP 70.790-075, Brasília-DF.

e-mail: universitas.saude@uniceub.br

Aos cuidados do Editor da revista.

Observe as Normas de Publicação. 